\title{
Serially Concatenated Unity-Rate Codes Improve Quantum Codes Without Coding-Rate Reduction
}

\author{
Zunaira Babar, Hung Viet Nguyen, Panagiotis Botsinis, Dimitrios Alanis, Daryus Chandra, Soon Xin Ng, \\ and Lajos Hanzo
}

\begin{abstract}
Inspired by the astounding performance of the unity rate code (URC) aided classical coding and detection schemes, we conceive a quantum URC (QURC) for assisting the design of concatenated quantum codes. Unfortunately, a QURC cannot be simultaneously recursive as well as non-catastrophic. However, we demonstrate that, despite being non-recursive, our proposed QURC yields efficient concatenated codes, which exhibit a low error rate and a beneficial interleaver gain, provided that the coding scheme is carefully designed with the aid of EXtrinsic Information Transfer (EXIT) charts.
\end{abstract}

Index Terms-Quantum error correction, turbo codes, EXIT charts, unity rate code.

Manuscript received April 21, 2016; revised June 18, 2016; accepted July 19, 2016. The financial support of the European Research Council under the Advanced Fellow Grant, that of the Royal Society's Wolfson Research Merit Award, and that of the Engineering and Physical Sciences Research Council under Grant EP/L018659/1 is gratefully acknowledged. The research data for this paper is available at []. The associate editor coordinating the review of this paper and approving it for publication was T. Ngatched.

The authors are with the School of Electronics and Computer Science, University of Southampton, Southampton SO17 1BJ, U.K. (e-mail: zb2g10@ecs.soton.ac.uk; hvn08r@ecs.soton.ac.uk; pb1y14@ecs.soton.ac.uk; da4g11@ecs.soton.ac.uk; dc2n14@ecs.soton.ac.uk; sxn@ecs.soton.ac.uk; 1h@ecs.soton.ac.uk).

Digital Object Identifier 10.1109/LCOMM.2016.2593874
It was also demonstrated in [6] that concatenated classical codes, relying on an outer Reed-Solomon code having an arbitrary coding rate, may asymptotically achieve the Gilbert-Varshamov bound, when a random rate-1 inner code is invoked. These results were recently extended to the quantum domain [7]. However, since QCCs cannot be simultaneously recursive as well as non-catastrophic, we have to compromise concerning one of these attributes, while designing a rate-1 QCC. A non-recursive inner code may result in a bounded minimum distance, while a catastrophic inner code would require code doping for supporting iterative decoding. It was demonstrated in [8] that a recursive catastrophic rate-1 QCC does not yield appreciable benefits in terms of the achievable Word Error Rate (WER). Despite having a so-called unbounded minimum distance, the design of [8] exhibits a high WER performance and was hence classified as an 'errorreducing' code rather than an error-correcting code. Motivated by these developments, in this letter we conceive a novel non-recursive and non-catastrophic Quantum Unity Rate Code (QURC), which may be used in combination with an arbitrary outer code for improving its performance without any codingrate reduction, while imposing only a modestly increased encoding complexity. We quantify the benefits of our proposed QURC with the aid of design examples. We demonstrate that the proposed QURC-aided concatenated scheme is capable of achieving a low WER and a high interleaver gain, when the code is carefully designed with the aid of EXIT charts.

This letter is organized as follows. In Section II-A, we detail our system model, followed by the portrayal of our proposed QURC in Section II-B. Our results are discussed in Section III, while our conclusions are offered in Section IV.

\section{QURC-Aided Concatenated Code Design}

\section{A. System Model}

Fig. 1 shows the schematic of a QURC-aided concatenated quantum code. The outer encoder $\mathcal{V}_{1}$ is an $[n, k, m]$ QCC, which encodes $k$ logical qubits (or information word) $\left|\psi_{1}\right\rangle$ into $n$ physical qubits (or codeword) $\left|\bar{\psi}_{1}\right\rangle$ with the aid of $(n-k)$ auxiliary qubits and $m$ memory qubits. The physical qubits $\left|\bar{\psi}_{1}\right\rangle$ are interleaved and fed to a QURC, which encodes the $n$ input logical qubits $\left|\psi_{2}\right\rangle$ into the $n$ physical qubits $\left|\bar{\psi}_{2}\right\rangle$ without any additional redundancy. QURC merely acts as a precoder invoked for supporting iterative decoding. The precoded stream $\left|\bar{\psi}_{2}\right\rangle$ is transmitted through a quantum depolarizing channel, which is a commonly used channel model [2], [3], [5]. Explicitly, a quantum depolarizing channel having a depolarizing probability $p$ inflicts an $n$-qubit Pauli error $\mathcal{P}_{2}$, 


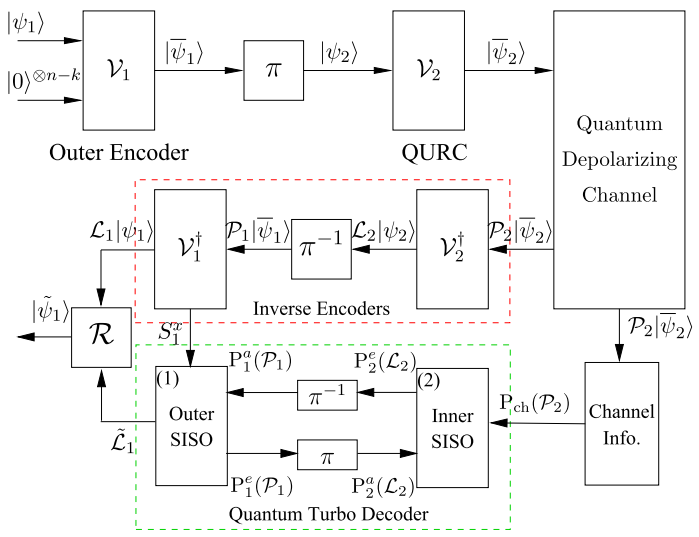

Fig. 1. Schematic of a QURC-aided concatenated quantum coding scheme. $\mathrm{P}_{i}^{a}($.$) and \mathrm{P}_{i}^{e}($.$) denote the a-priori and extrinsic probabilities related to the$ ith decoder.

where a qubit may independently experience either a bit-flip or a phase-flip or in fact both, each with a probability of $p / 3$.

The received physical information $\left|\hat{\psi}_{2}\right\rangle=\mathcal{P}_{2}\left|\bar{\psi}_{2}\right\rangle$ is processed by the serially concatenated inverse encoders ${ }^{1} \mathcal{V}_{i}^{\dagger}$ of Fig. 1, resulting in the potentially erroneous logical qubits $\mathcal{L}_{i}|\psi\rangle$ as well as the erroneous syndrome qubits $\mathcal{S}_{1}|\psi\rangle$. The latter collapse to the classical bits $S_{1}^{x}$ upon measurement. Using the classical syndrome $S_{1}^{x}$ and the channel information $\mathrm{P}_{\mathrm{ch}}\left(\mathscr{P}_{2}\right)$, the pair of serially concatenated syndrome-based classic Soft-In Soft-Out (SISO) decoders of Fig. 1 engage in degenerate iterative decoding [2], [3] for estimating the most likely error coset $\tilde{\mathcal{L}}_{1}$ imposed on the logical qubits of the outer decoder. We emphasize that in contrast to the classic SISO decoder, the syndrome-based SISO decoder generates extrinsic information pertaining to the logical error $\mathcal{L}_{i}$ and the physical error $\mathcal{P}_{i}$. Finally, the recovery operation $\mathcal{R}$ is applied to the erroneous logical qubits $\mathcal{L}_{1}\left|\psi_{1}\right\rangle$ based on the estimated error $\tilde{L}_{1}$, hence yielding the recovered output $\left|\tilde{\psi}_{1}\right\rangle$.

The convergence behaviour of the iterative decoder of Fig. 1 may be visualized using EXIT charts [4], which model the flow of information between the concatenated syndrome-based decoders. Explicitly, an EXIT chart characterizes the average Mutual Information (MI) transfer characteristics of the inner and outer decoders in terms of: the a-priori MI $I_{A}\left(L_{2}\right)$ between $\mathcal{L}_{2}$ and $\mathbf{P}_{2}^{a}\left(\mathcal{L}_{2}\right)$, the extrinsic MI $I_{E}\left(\mathcal{L}_{2}\right)$ between $\mathcal{L}_{2}$ and $\mathbf{P}_{2}^{e}\left(\mathcal{L}_{2}\right)$, the a-priori MI $I_{A}\left(\mathcal{P}_{1}\right)$ between $\mathcal{P}_{1}$ and $\mathbf{P}_{1}^{a}\left(\mathcal{P}_{1}\right)$, as well as the the extrinsic MI $I_{E}\left(\mathcal{P}_{1}\right)$ between $\mathcal{P}_{1}$ and $\mathbf{P}_{1}^{e}\left(\mathcal{P}_{1}\right)$. The a-priori information of both decoders is modeled using an independent Gaussian distribution, while the output extrinsic MI can be computed using [9]:

$$
I_{E}(\mathcal{A})=\frac{1}{2}\left(2+\mathrm{E}\left[\sum_{\mathrm{m}=0}^{3} \mathbf{P}_{2}^{e}\left(\mathcal{A}^{j(\mathrm{~m})}\right) \log _{2} \mathbf{P}_{2}^{e}\left(\mathcal{A}^{j(\mathrm{~m})}\right)\right]\right),
$$

where $\mathrm{E}$ is the expectation (or time average) operator, while $\mathcal{A}^{j(\mathrm{~m})}$ is equivalent to the $\mathrm{m}^{\text {th }}$ quaternary hypothetical error (no-error, bit-flip, phase-flip or bit-phase-flip) imposed on the $j^{\text {th }}$ logical/physical qubit of the inner/outer decoder. The resultant inner decoder's EXIT function $T_{2}$ and the outer

\footnotetext{
${ }^{1}$ An inverse encoder maps physical qubits onto the logical qubits.
}

decoder's EXIT function $T_{1}$ is given by:

$$
I_{E}\left(\mathcal{L}_{2}\right)=T_{2}\left[I_{A}\left(\mathcal{L}_{2}\right), p\right], \quad I_{E}\left(\mathcal{P}_{1}\right)=T_{1}\left[I_{A}\left(\mathcal{P}_{1}\right)\right] .
$$

Finally, the EXIT functions of Eq. (2) are plotted in the same graph, with the $x$ and $y$ axes of the outer decoder swapped. Hence, the exchange of MI between the two decoders may be visualized as a stair-case-shaped decoding trajectory in the resultant EXIT chart. For the sake of approaching the achievable capacity, it is desirable to create a marginally open tunnel between the inner and outer decoders' EXIT curves at the highest possible depolarizing probability [4].

\section{B. Proposed Quantum Unity Rate Code}

The encoders of Fig. 1 are stabilizer codes [5], [10], which belong to the family of Clifford operators [11]. A distinct feature of the Clifford family is that they preserve the elements of the Pauli group under conjugation. More explicitly, for an $n$-qubit Pauli operator $\mathcal{P}$, which belongs to the Pauli group $\mathcal{G}_{n}$, an $n$-qubit Clifford operator $\mathcal{V}$ ensures that $\mathcal{V} \mathcal{P} \mathcal{V}^{\dagger} \in G_{n}$ [11]. Consequently, the stabilizer generators of the encoder $\mathcal{V}$ are intrinsically commutative. Such an encoder $\mathcal{V}$ may be realized using the Hadamard $(\mathbf{H})$, phase $(\mathbf{S})$ and controlled-NOT (C-NOT) gates, which are defined as [5]:

$$
\mathbf{H}=\frac{1}{\sqrt{2}}\left(\begin{array}{cc}
1 & 1 \\
1 & -1
\end{array}\right), \mathbf{S}=\left(\begin{array}{ll}
1 & 0 \\
0 & i
\end{array}\right), \mathrm{C}-\mathrm{NOT}=\left(\begin{array}{llll}
1 & 0 & 0 & 0 \\
0 & 1 & 0 & 0 \\
0 & 0 & 0 & 1 \\
0 & 0 & 1 & 0
\end{array}\right)
$$

Furthermore, the Clifford encoder $\mathcal{V}$ of an $[n, k, m]$ QCC consists of concatenated $(n+m)$-qubit sub-encoders $\mathcal{U}$, conventionally termed as 'seed transformations', so that the adjacent sub-encoders have an overlap of $m$ memory qubits.

For the sake of finding an optimized QURC construction, we randomly constructed Clifford seed transformations $\mathcal{U}$ using the classical random walk algorithm over the $(1+m)$-qubit Clifford group as in [12] and analyzed the associated inner EXIT curves at an arbitrarily chosen depolarizing probability of $p=0.07$. We restricted our search space to $m=1$ and $m=2$ for achieving a low-complexity design, since the decoding complexity increases exponentially with the number of memory states. Furthermore, ideally, it is desired that the inner component of a concatenated code should be both recursive as well as non-catastrophic. However, QCCs cannot be recursive and non-catastrophic at the same time [3]. A noncatastrophic behaviour permits iterative decoding convergence, while having a recursive structure is usually desired for a beneficial interleaver gain, hence resulting in a Quantum Bit Error Rate (QBER) reduction upon increasing the frame length, which asymptotically tends zero. Consequently, a catastrophic QURC does not yield non-trivial extrinsic information in the absence of a-priori information, while its recursive structure ensures that its inner EXIT curve reaches the $(1,1)$-point of perfect convergence. This is demonstrated in Fig. 2, where the inner EXIT curve of a catastrophic and recursive QURC is plotted. Since the resultant inner EXIT curve emerges from the $(0,0)$-point, code doping is required for initiating the iterative decoding process. Therefore, we focus our attention on designing a non-catastrophic and non-recursive QURC. 


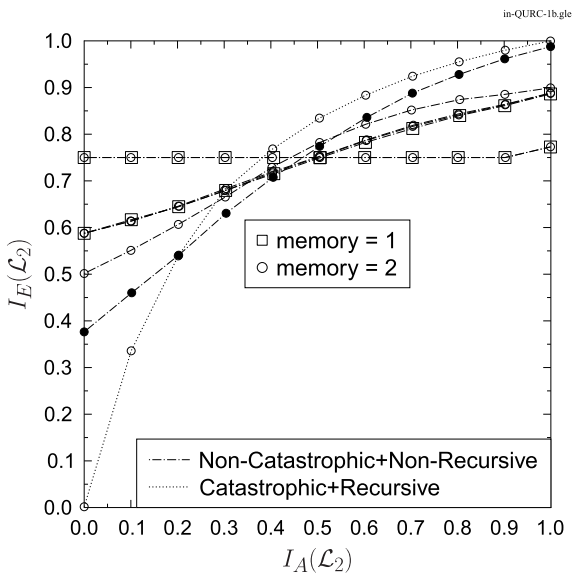

Fig. 2. Inner EXIT curves of randomly constructed non-catastrophic and non-recursive QURCs at $p=0.07$. The proposed QURC is represented by 'filled circles'. A catastrophic and recursive QURC is also featured as an example.

Fig. 2 shows the inner EXIT curves of the various noncatastrophic and non-recursive QURCs, relying on randomly constructed unique seed transformations. We may observe in Fig. 2 that the inner EXIT curve of non-catastrophic and non-recursive QURCs starts from the $(0, y)$-point for $y>0$ and terminates at the $(1, y)$-point for $y<1$. For such inner EXIT curves, we can still achieve decoding convergence to an infinitesimally low QBER upon increasing the frame length, provided that the outer decoder's EXIT curve only crosses the inner decoder's EXIT curve close to the $x=1$ line. Intuitively, if the outer decoder's EXIT curve does not intersect with that of the inner code before reaching the $x=1$ line, then the QBER may asymptotically approach zero for an infinite frame length. Hence, by carefully matching the outer decoder's EXIT curve to that of the inner decoder, we may maximize the interleaver gain. To facilitate this curvematching for a wider range of outer codes, it is desirable that the inner decoder's EXIT curve should terminate close to the $(1,1)$-point. Therefore, we select the inner EXIT curve marked by 'filled circles' in Fig. 2, whose seed transformation is:

$$
\mathcal{U}=\{21,56,5,46,44,38\}_{\text {decimal }} .
$$

\section{Results AND Discussions}

For the sake of quantifying the explicit benefit of our proposed QURC, we designed a 1/2 rate concatenated code using the 10-subcode QIRCC of [5] as the outer component. Explicitly, the QIRCC of [5] consists of five strong memory-3 QCCs followed by five weak memory-1 QCCs, having the coding rates of $[1 / 4,1 / 3,1 / 2,2 / 3,3 / 4]$. The weighting coefficients of the QIRCC were optimized using the optimization algorithm discussed in [5] for ensuring that the resultant outer decoder's EXIT curve closely matched the inner decoder's EXIT curve at the highest possible depolarizing probability. It was found that using the

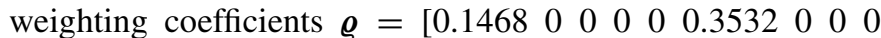
$0.5]^{T}$, the QIRCC would yield a marginally open EXIT chart tunnel at $p=0.06$, as shown in Fig. 3. Observe in Fig. 3 that the inner and outer decoder's EXIT curves crossover before

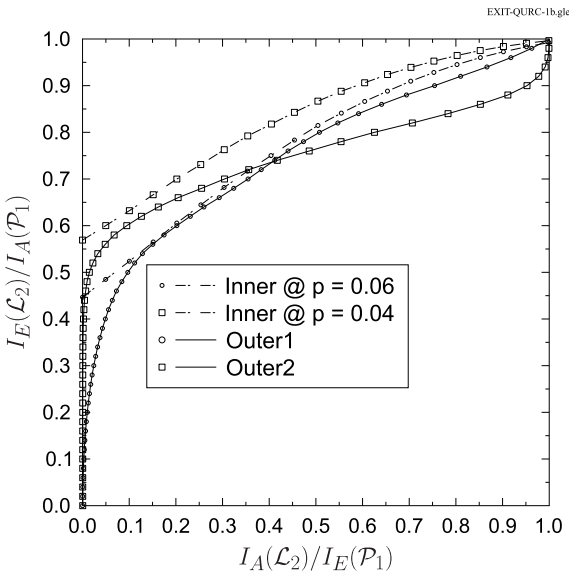

Fig. 3. EXIT-curve matching for the QURC-QIRCC scheme. The labels 'Outer1' and 'Outer2' represent the 10-subcode and 5-subcode QIRCC, respectively.

reaching the $x=1$ line, which is expected to result in a low interleaver gain as well as a high error floor.

To prove further, both the WER as well as QBER performance of the resultant QURC-QIRCC scheme is quantified in Fig. 4a upon increasing the frame length. As predicted by the EXIT chart of Fig. 3, the QBER improves upon increasing the frame length for $p<0.06$, albeit rather slowly. Furthermore, the WER degrades upon increasing the frame length. This is due to a pair of counteracting forces - the improved interleaver gain reduces the QBER upon increasing the frame length, while the WER is increased with the frame length. Hence, since the interleaver gain improvement remains modest, the resultant WER is ultimately degraded upon increasing the frame size.

For ensuring a significant interleaver gain, the inner and outer decoder's EXIT curves should not intersect before reaching the $x=1$ line. A crossover may be prevented by using an outer code having a large minimum distance. Therefore, as another example, we consider a 5-subcode QIRCC, which only employs the five strong memory- 3 components of the 10 -subcode QIRCC. It was found that the weighting coefficients $\varrho=\left[\begin{array}{llllll}0.1316 & 0.1869 & 0.2962 & 0.3852 & 0\end{array}\right]^{T}$ yield a narrow open EXIT-chart tunnel at $p=0.04$, as shown in Fig. 3 . The convergence threshold of $p=0.04$ is lower than that of Fig. 4, yet the inner and outer EXIT curves do not crossover. The resultant WER/QBER performance is recorded in Fig. 4b, both of which improve upon increasing the frame length.

In Table I, we have benchmarked the performance of our designed QURC-QIRCC scheme $(N=2000)$ of Fig. $4 b$, at a WER of $10^{-3}$ against the best known iterative schemes, which have a similar coding rate. More specifically, we have considered the Spatially-Coupled Quasi-Cyclic Quantum Low Density Parity Check (SC QC-QLDPC) code of [13] having a coding rate of 0.49 and a length of $n=181000$ and the nonbinary QC-QLDPC code of [14] having a coding rate of 0.5 , a length of $n=8768$ and a Galois field of $\operatorname{GF}\left(2^{8}\right)$. It may be observed in Table I that the depolarizing probability achievable by the SC QC-QLDPC code at a WER of $10^{-3}$ is about $9 \%$ lower than our QURC-QIRCC concatenated scheme, while that of the non-binary QC-QLDPC code is about $15 \%$ higher. 


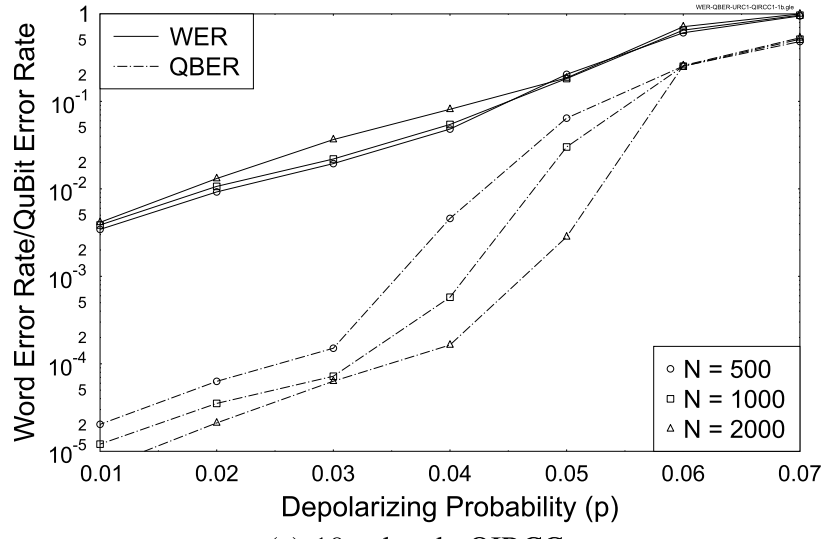

(a) 10-subcode QIRCC.

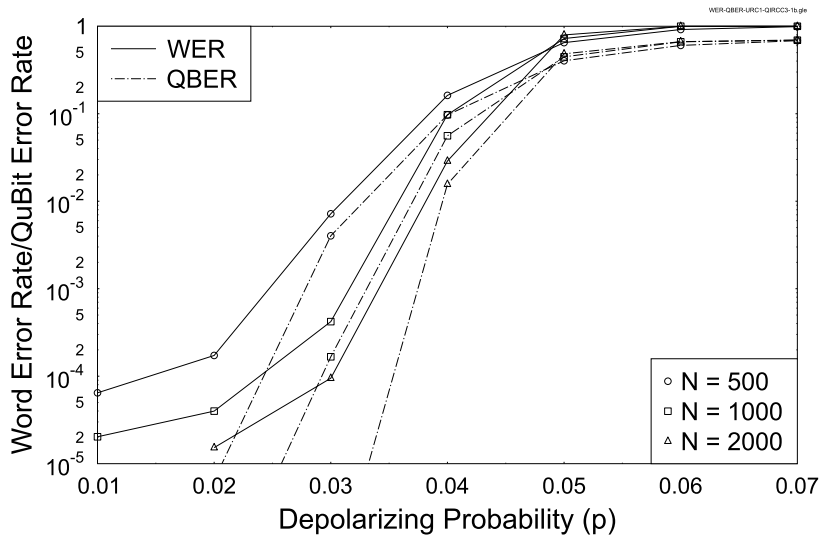

(b) 5-subcode QIRCC.

Fig. 4. Achievable WER and QBER performance of the 1/2-rate QURC-QIRCC schemes, when a maximum of 20 decoding iterations are invoked. $N$ denotes the number of input logical qubits per frame.

TABLE I

AChievable Depolarizing Probability $\left(p_{i}\right)$ AT a WER of $10^{-3}$

\begin{tabular}{|c|l|l|l|}
\hline No. & Coding Scheme & $p_{i}$ & Comparison \\
\hline 1 & $\begin{array}{l}\text { QURC-QIRCC of Fig. 4b }(N=2000), \\
R=0.5\end{array}$ & $p_{1}=0.034$ & 1 \\
2 & SC QC-QLDPC of [13], $R=0.49$ & $p_{2}=0.031$ & $\frac{p_{2}}{p_{1}}=0.91$ \\
3 & Non-binary QC-QLDPC of [14], $R=0.5$ & $p_{3}=0.039$ & $\frac{p_{3}}{p_{1}}=1.15$ \\
\hline
\end{tabular}

The improved performance of the non-binary QC-QLDPC code is achieved at the expense of a longer transmission frame and a higher decoding complexity.

\section{Conclusions}

In this letter, we have conceived a QURC-aided concatenated quantum code design, which relies on a non-recursive and non-catastrophic QURC for improving the performance of the outer code without any coding-rate reduction. We have demonstrated with the aid of design examples that efficient concatenated quantum codes, relying on a non-recursive QURC, may be designed with the aid of EXIT charts. We have also benchmarked the performance of our designed QURCaided scheme against the best known QLDPC codes.

\section{REFERENCES}

[1] L. L. Hanzo, T. H. Liew, B. L. Yeap, R. Y. S. Tee, and S. X. Ng, Turbo Coding, Turbo Equalisation and Space-Time Coding: EXIT-Chart-Aided Near-Capacity Designs for Wireless Channels, 2nd ed. New York, NY, USA: Wiley, Mar. 2011.
[2] D. Poulin, J.-P. Tillich, and H. Ollivier, "Quantum serial turbo codes," IEEE Trans. Inf. Theory, vol. 55, no. 6, pp. 2776-2798, Jun. 2009.

[3] M. M. Wilde, M.-H. Hsieh, and Z. Babar, "Entanglement-assisted quantum turbo codes," IEEE Trans. Inf. Theory, vol. 60, no. 2 pp. 1203-1222, Feb. 2014.

[4] Z. Babar, S. X. Ng, and L. Hanzo, "EXIT-chart-aided near-capacity quantum turbo code design," IEEE Trans. Veh. Technol., vol. 64, no. 3, pp. 866-875, Mar. 2015.

[5] Z. Babar, P. Botsinis, D. Alanis, S. X. Ng, and L. Hanzo, "The road from classical to quantum codes: A hashing bound approaching design procedure," IEEE Access, vol. 3, pp. 146-176, 2015.

[6] C. Thommesen, "The existence of binary linear concatenated codes with Reed-Solomon outer codes which asymptotically meet the GilbertVarshamov bound," IEEE Trans. Inf. Theory, vol. 29, no. 6, pp. 850-853, Nov. 1983.

[7] Y. Ouyang, "Concatenated quantum codes can attain the quantum Gilbert-Varshamov bound," IEEE Trans. Inf. Theory, vol. 60, no. 6 , pp. 3117-3122, Jun. 2014.

[8] M. Abbara and J.-P. Tillich, "Quantum turbo codes with unbounded minimum distance and excellent error-reducing performance," in Proc IEEE Inf. Theory Workshop (ITW), Oct. 2011, pp. 252-256.

[9] J. Kliewer, S. X. Ng, and L. Hanzo, "Efficient computation of EXIT functions for nonbinary iterative decoding," IEEE Trans. Commun., vol. 54, no. 12, pp. 2133-2136, Dec. 2006.

[10] Y. Xie, L. Yang, and J. Yuan, "q-ary chain-containing quantum synchronizable codes," IEEE Commun. Lett., vol. 20, no. 3, pp. 414-417, Mar. 2016.

[11] J. Dehaene and B. De Moor, "Clifford group, stabilizer states, and linear and quadratic operations over GF(2)," Phys. Rev. A, vol. 68, p. 042318, Oct. 2003. [Online]. Available: http://link.aps. org/doi/10.1103/PhysRevA.68.042318

[12] D. P. DiVincenzo, D. W. Leung, and B. M. Terhal, "Quantum data hiding," IEEE Trans. Inf. Theory, vol. 48, no. 3, pp. 580-598, Mar. 2002.

[13] M. Hagiwara, K. Kasai, H. Imai, and K. Sakaniwa, "Spatially coupled quasi-cyclic quantum LDPC codes," in Proc. IEEE Int. Symp. Inf. Theory Process., Jul./Aug. 2011, pp. 638-642.

[14] K. Kasai, M. Hagiwara, H. Imai, and K. Sakaniwa, "Quantum error correction beyond the bounded distance decoding limit," IEEE Trans. Inf. Theory, vol. 58, no. 2, pp. 1223-1230, Feb. 2012. 


\section{AUTHOR QUERIES}

\section{AUTHOR PLEASE ANSWER ALL QUERIES}

PLEASE NOTE: We cannot accept new source files as corrections for your paper. If possible, please annotate the PDF proof we have sent you with your corrections and upload it via the Author Gateway. Alternatively, you may send us your corrections in list format. You may also upload revised graphics via the Author Gateway.

AQ:1 = Please be advised that per instructions from the Communications Society this proof was formatted in Times Roman font and therefore some of the fonts will appear different from the fonts in your originally submitted manuscript. For instance, the math calligraphy font may appear different due to usage of the usepackage[mathcal]euscript. We are no longer permitted to use Computer Modern fonts. $\mathrm{AQ}: 2$ = Please confirm/give details of funding source.

AQ:3 = Please provide the complete sentence for "The research data for this paper..." in first footnote.

AQ:4 = Note that if you require corrections/changes to tables or figures, you must supply the revised files, as these items are not edited for you. 